\title{
Saúde, meio ambiente e segurança do trabalho associado ao uso de agrotóxicos
}

\section{Health, environment and safety associated with the use of pesticides}

\author{
Luara Lourenço Ismael ${ }^{1}$, Hozana Raquel de Medeiros Garcia ${ }^{2}$, Wanessa Alves Martins ${ }^{3}$, Jose Augusto ${ }^{4}$
}

\begin{abstract}
RESUMO - O trabalhador rural está exposto a diferentes situações de riscos durante o desenvolvimento de suas atividades no campo. Uma delas refere-se ao manuseio e aplicação incorreta de agrotóxicos, o que acaba por provocar sérios danos á saúde, não apenas do próprio agricultor mais da população local por consumir alimentos contaminados e ao ambiente. Para isto, o objetivo geral dessa pesquisa foi realizar um levantamento bibliográfico acerca dos riscos advindos das atividades agrícolas quanto ao uso de agrotóxicos. Os resultados evidenciaram que grande parte dos acidentes de trabalho, estão associados à utilização incorreta dos EPIs, além da falta de informação e treinamento dos agricultores para desenvolver tais atividades. Nesse contexto, medidas de caráter preventivo e corretivo deverão ser tomadas para a redução do uso e exposição aos agrotóxicos, para isso, devem ser implementadas no ambiente de trabalho ações socioeconômicas, políticas e ambientais.
\end{abstract}

Palavras-chaves - Trabalhador Rural, EPIs, Desenvolvimento Sustentável.

ABSTRACT - The rural worker is exposed to different risk situations while developing their activities in the field. One of them refers to the incorrect handling and application of pesticides, which ultimately cause serious damage to health, not just the very best of the local population farmer by consuming contaminated food and the environment. For this, the general objective of this research was to conduct a literature review about the risks arising from agricultural activities on the use of pesticides. The results showed that most industrial accidents are related to improper use of PPE, as well as lack of information and training of farmers to develop such activities. In this context, preventive and corrective nature of measures should be taken to reduce the use and exposure to pesticides, so it should be implemented in the workplace actions socioeconomic, political and environmental.

Key words - Farm Worker, PPE, Sustainable Development.

\footnotetext{
*Autor para correspondência

Recebido para publicação em 13/10/2015; aprovado em 15/12/2015

${ }^{1}$ Engenheira Ambiental (UFCG), Mestranda Pós -Graduação Eng. Civil e Ambiental - UFPB, (83) 9831-1665, luara_ismael@hotmail.com

${ }^{2}$ Gestora Ambiental (UERN), Mestranda Pós -Graduação Eng. Civil e Ambiental - UFPB, hozana_raquel@hotmail.com

${ }^{3}$ Engenheira Ambiental (UFCG), Mestranda Pós -Graduação Eng. Civil e Ambiental - UFPB, wanessa_ufcg@hotmail.com

${ }^{4}$ Tecnólogo em Agroecologia (IFPB), Mestrando Pós -Graduação Eng. Civil e Ambiental - UFPB, jsaugusto.eco@gmail.com
} 


\section{INTRODUÇÃO}

A utilização dos agrotóxicos no meio rural brasileiro vem acarretando uma série de consequências, tanto para a saúde do trabalhador como para o ambiente. Em geral, tais implicações são resultantes do uso inadequado dessas substâncias, da pressão exercida pela indústria e o comércio, da alta toxicidade de certos produtos, da ausência de informações a respeito da saúde e segurança dos trabalhadores e da precariedade dos mecanismos de vigilância (MONQUERO et al., 2009).

Os agrotóxicos quando utilizados em concentrações que ultrapassam os limites permitidos podem representar perigo para a vida animal, incluindo os seres humanos. Sua aplicação contamina os solos, a água e o ar, e a exposição constante pode causar sérios problemas crônicos (DOMINGUES, 2010).

De acordo com a Norma Regulamentadora (NR-31), que trata da segurança e saúde no trabalho na agricultura, pecuária, silvicultura, exploração florestal e aquicultura os empregadores rurais devem implementar ações de segurança e saúde que visem a prevenção de acidentes e doenças decorrentes do trabalho na unidade de produção rural, atendendo a seguinte ordem de prioridade: eliminação de riscos através da substituição ou adequação dos processos produtivos, máquinas e equipamentos; adoção de medidas de proteção coletiva para controle dos riscos na fonte e adoção de medidas de proteção pessoal.

O manuseio inadequado de produtos tóxicos é um dos principais causadores de problemas de saúde no campo. Os trabalhadores rurais que utilizam de tais práticas estão sujeitos a sofrerem seriamente com problemas de saúde. No Brasil, segundo o Sistema Nacional de Informações Toxicológicas SINITOX foi notificado no ano de 2009 aproximadamente um total de 11.641 casos de intoxicações agudas por agrotóxicos de uso agrícola, número que pode ser subestimado considerando-se a elevada subnotificação desses eventos no País (CORDEIRO, 2014). Para minimizar a exposição aos riscos é fundamental adotar práticas de biossegurança, como por exemplo, utilizar corretamente os Equipamentos de Proteção Individual-EPIs.

No Brasil, o problema de inadequação dos EPIs às condições ergonômicas e ambientais também é comum na grande maioria das atividades. Na agricultura, por exemplo, especialmente em pequenas comunidades rurais, é comum deparar-se com trabalhadores rurais sem utilizar os EPIs obrigatórios durante a manipulação e a aplicação de agrotóxicos (VEIGA, et al., 2007; SALEM E MOURA, 2014).

O emprego de EPIs, apesar de não desejado por muitos dos trabalhadores, deve ser considerado como tecnologia de proteção disponível dentro de uma visão integrada e sistêmica de abordagem dos problemas ocupacionais (VEIGA, et al.,2007).

Assim sendo, a presente pesquisa tem por objetivo principal identificar e caracterizar os riscos advindos das atividades associadas ao uso de agrotóxicos para o trabalhador rural e para o meio ambiente. Para tanto a investigação propõe discutir sobre as implicações do uso destas substancias para a saúde do trabalhador rural quanto ao uso inadequado dos EPIs, apresentando soluções preventivas sob a problemática a ser investigada com base nas normas regulamentadoras pertinentes.

\section{MATERIAL E MÉTODOS}

O presente estudo utilizou o método de pesquisa qualitativo, empregando uma abordagem descritiva na qual visa investigar algo que acontece baseando-se em observações, como por exemplo, levantamentos documentais (APPOLINÁRIO, 2006).

A revisão de literatura foi realizada por meio de consultas a acervos bibliográficos publicados em periódicos de relevância, dissertações, teses, livros e consultas a dados disponíveis em endereços eletrônicos, como por exemplo, Agência Nacional de Vigilância Sanitária (ANVISA), Ministério do Trabalho e Emprego, Ministério da Agricultura, Pecuária e Abastecimento (MAPA), Empresa Brasileira de Pesquisa Agropecuária (EMBRAPA), dentre outros de interesse.

\section{REVISÃO BIBLIOGRÁFICA}

\section{Agrotóxicos: legislação e impactos ao ambiente e a saúde do trabalhador rural}

No Brasil os produtos químicos utilizados para o controle de pragas no campo ganharam legislação específica em 1989, com a Lei Federal $n^{\circ} 7.802$, regulamentada pelo Decreto $n^{\circ} 4074$ de 04/01/2002, a qual define os agrotóxicos e afins como sendo os produtos e elementos de processos químicos, físicos ou biológicos remetidos ao uso nos setores de produção, armazenamento e beneficiamento de produtos agrícolas, nas pastagens, na proteção de florestas nativas ou implantadas e em outros ecossistemas, além de ambientes urbanos, hídricos e industriais; cujo propósito seja alterar a composição da fauna e flora, com o intuito de preservá-las da ação danosa de seres vivos considerados nocivos (BRASIL, 1989).

Dentre as vantagens observadas com o uso dos agrotóxicos destaca-se o controle eficaz de pragas e de outros elementos nocivos às plantações; aumento da produção de alimentos, suprindo assim grande parte da demanda mundial (VIGNA, 2010). Quando utilizados incorretamente, os agrotóxicos podem provocar contaminações nos trabalhadores rurais responsáveis pela aplicação, nos consumidores de alimentos, nos animais e no ambiente (BARROSO E WOLFF, 2009).

Segundo a Agência Nacional de Vigilância Sanitária ANVISA (2012), em 2008 o Brasil alcançou o posto de primeiro lugar no ranking de maior consumidor de agrotóxicos do mundo, apresentando um crescimento no mercado nacional de $190 \%$ comparado com o crescimento do mercado mundial de $93 \%$.

A exposição humana aos agrotóxicos tem se configurado um sério problema de saúde pública. Os trabalhadores rurais durante toda sua jornada de trabalho necessitam de proteção e cuidado com sua saúde e de informações básicas sobre os riscos inerentes ao uso de agrotóxicos.

De acordo com Silva et al., (2005), além da exposição ocupacional, a contaminação alimentar e ambiental coloca em risco de intoxicação outros grupos populacionais, com destaque para as famílias dos agricultores, a população 
circunvizinha a uma unidade produtiva e a população em geral, que se alimenta do que é produzido no campo.

A contaminação ocupacional pelos agrotóxicos pode ser observada nas etapas: aquisição, transporte, armazenamento, manuseio (principalmente no preparo da calda), aplicação e disposição final de sobras e de embalagens vazias (BARROSO E WOLFF, 2009). Apesar de atingir uma pequena parcela da população (os trabalhadores rurais e guardas de endemias) o processo de manipulação dos agrotóxicos é a via responsável por mais de $80 \%$ dos casos de intoxicação, levando em consideração a intensidade e a frequência de contato entre este grupo populacional e o produto (PINHEIRO E ADISSI, 2007).

Os agrotóxicos são absorvidos pelo corpo humano pelas vias respiratórias e dérmicas e, em menor quantidade, também pela via oral. Uma vez no organismo humano, poderão causar quadros de intoxicação aguda ou crônica (SILVA et al., 2005). No primeiro caso, os sintomas manifestam-se mais rapidamente no organismo, causando dores de cabeça, dores de estômago, sonolência, tontura, fraqueza, perturbação da visão, saliva e suor excessivos, dificuldade respiratória e diarreia. Na forma crônica, os efeitos da intoxicação podem surgir meses ou até anos depois da exposição ao produto (MONQUERO, et al., 2009).

Cabe destacar que as contaminações podem ser evitadas com hábitos simples de higiene. Via de regra, os casos de contaminação são resultado de erros cometidos durante as etapas de manuseio ou aplicação de produtos fitossanitários e são causados na maioria das vezes pela falta de informação ou simples displicência do operador (IWAMI, et al., 2008).

\section{Segurança do trabalho aplicada ao uso de agrotóxicos}

As ações de segurança e saúde no ambiente rural devem contemplar os seguintes aspectos: melhoria das condições e do meio ambiente de trabalho; promoção da saúde e da integridade física dos trabalhadores rurais; campanhas educativas de prevenção de acidentes e doenças decorrentes do trabalho (BRASIL, 2005).

O uso inadequado dos EPIs e até mesmo a não utilização dos mesmos durante o manuseio com substancias químicas na agricultura, reflete uma problemática bastante discutida nos dias atuais, sendo de fundamental importância investigar quais as implicações de tais práticas principalmente para a saúde do trabalhador rural.

Os EPIs quando utilizados na aplicação de agrotóxicos, são projetados de forma a garantir proteção contra agentes químicos externos, ou seja, para manter certas substâncias "fora" do organismo. As mesmas propriedades físicas e químicas que fornecem aos EPIs essa característica de proteção também os transformam, frequentemente, em bastante desconfortáveis e/ou inadequados. Esse desconforto no uso pode tornar a utilização de alguns EPIs um verdadeiro incômodo (VEIGA et al., 2007).

Segundo Azevedo e Freire (2006) os principais EPIs indicados para manuseio e aplicação de agrotóxicos são: Luvas; Respiradores; Viseira Facial; Jaleco e Calça hidrorepelente; Jaleco e Calça em não-tecido; Boné árabe; Capuz ou toca; Avental; Botas.

Segundo Barroso e Wolff (2009) o simples fornecimento dos EPIs não garante a proteção da saúde do trabalhador e nem evita contaminações. Se utilizados incorretamente os
EPIs podem comprometer ainda mais a segurança do trabalhador, assim sendo, a percepção do risco aliado a um conjunto de informações e regras básicas de segurança são as ferramentas mais importantes para evitar a exposição e assegurar o sucesso das medidas individuais de proteção.

Em sua pesquisa Lins Filho (2013), buscando correlacionar os resultados do monitoramento do Programa de Análises de Resíduos de Agrotóxicos em Alimentos - PARA na Paraíba, com ênfase na cultura do abacaxi, observou por meio da aplicação de questionários estruturados em uma cooperativa de agricultores - COOPAGRO, que o manuseio e aplicação dos agrotóxicos era feito com irregularidades, como por exemplos: o uso de produtos não autorizados para a cultura do abacaxi, sendo estes permitidos para a cultura de cana-de-açúcar; a utilização parcial dos EPIs; e o descarte incorreto das embalagens vazias no ambiente.

Lins Filho (2013), ao analisar as respostas quanto ao uso de EPIs, observou que a maioria dos entrevistados (53\%), disseram não utiliza-los, pelo fato de estar sobre um clima muito quente, o que dificulta na pratica o uso das máscaras que é o principal EPI na proteção contra a inalação dos produtos. Na Figura 1. observa-se os percentuais obtidos através da aplicação dos questionários quanto ao uso dos EPIs.

Figura 1. Uso de EPIs entre os produtores de abacaxi da cooperativa COOPAGRO

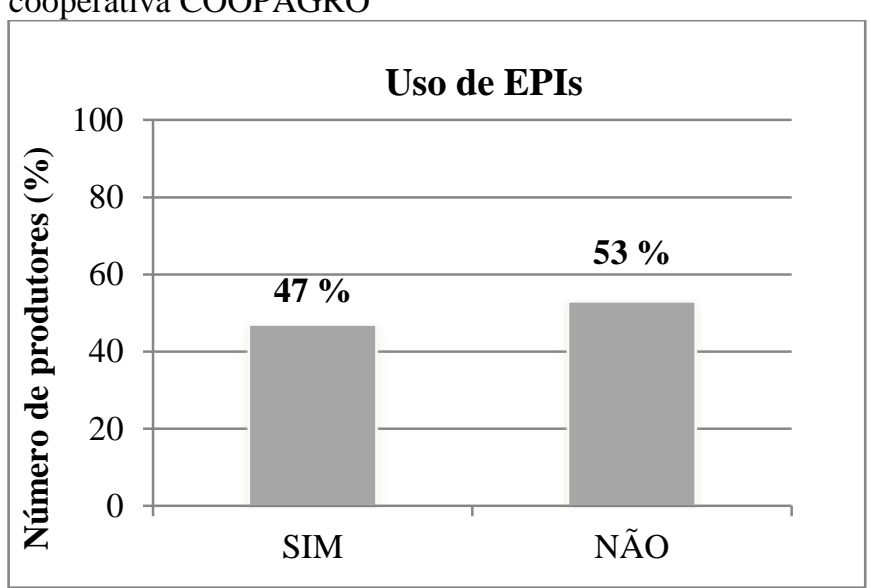

Fonte: Lins Filho (2013)

\section{Análise dos riscos que trabalhadores rurais estão expostos com o uso de agrotóxicos}

Nas atividades agrícolas, muitos fatores influenciam nas condições de saúde e segurança do trabalhador e, consequentemente, no aumento da gravidade dos riscos ocupacionais e ambientais, como por exemplo: os fatores climáticos (chuva, vento, raios ultravioletas), as condições do solo, os tipos de culturas, uso de produtos fitossanitários, problemas posturais, níveis de ruído acima do recomendado, às condições de moradias, higiene e outros.

Em sua pesquisa Waichman (2008), propôs uma avaliação integrada dos riscos do uso de agrotóxicos no estado do Amazonas e subdividiu esse processo de avaliação em três etapas como se observa na Figura 2. 
Figura 2. Esquema com as fases de avaliação integrada dos riscos ao uso de agrotóxicos.

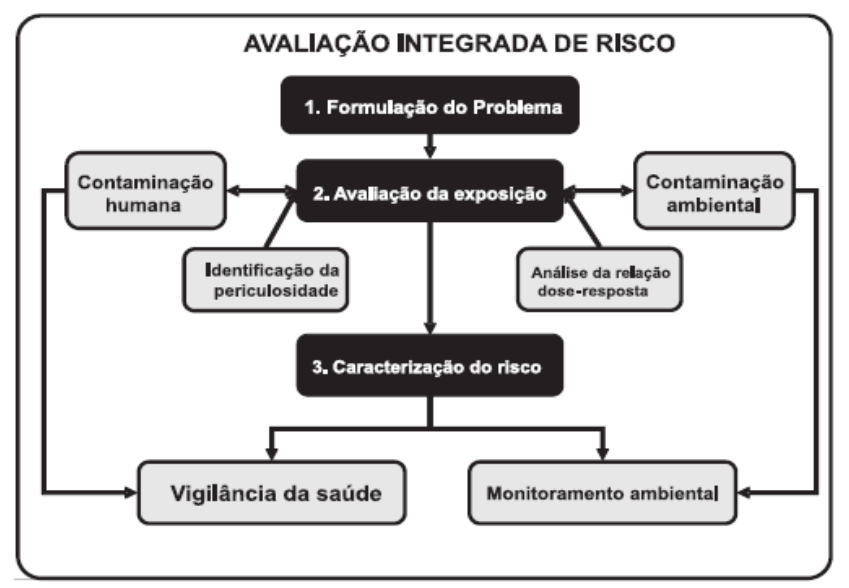

Fonte: Waichman (2008).
De acordo com a Figura 2, constata-se que a etapa inicial de avaliação integrada dos riscos é a formulação do problema o que permite, portanto, realizar a avaliação de exposição às concentrações dos agrotóxicos nos compartimentos bióticos e abióticos do ecossistema, posteriormente é possível fazer a caracterização do risco, que irá depender da análise dos pesticidas que são utilizados, a frequência e os locais de aplicação (WAICHMAN, 2008).

Uma etapa posterior à avaliação da exposição e considerada de suma importância é o manejo dos riscos. Nesse processo, são determinadas as medidas que deverão ser tomadas para a sua redução, para isso, devem ser consideradas ações ou medidas socioeconômicas e políticas de redução do uso e exposição de agrotóxicos ou opções alternativas a sua utilização (WAICHMAN, 2008).

Na Tabela 1 estão descritos de acordo com Salem e Moura (2014) e Abreu et al., (2011), os riscos que os trabalhadores rurais estão expostos nas atividades que envolvem o uso de agrotóxicos.

Tabela 1. Riscos que os trabalhadores rurais estão expostos nas atividades de manuseio e aplicação de agrotóxicos.

\begin{tabular}{|c|c|c|}
\hline $\begin{array}{l}\text { TIPO DE } \\
\text { RISCO }\end{array}$ & $\begin{array}{l}\text { FATOR DE } \\
\text { RISCO }\end{array}$ & AGRAVO OU DANO PARA A SAÚDE \\
\hline \multirow{4}{*}{ FÍSICOS } & Temperatura & $\begin{array}{l}\text { Estresse térmico, cãibras, síncope pelo calor, fadiga, } \\
\text { insolação. }\end{array}$ \\
\hline & $\begin{array}{l}\text { Frio, Vento e } \\
\text { Chuva }\end{array}$ & $\begin{array}{l}\text { Afecções de vias áreas superiores, } \\
\text { resfriado. }\end{array}$ \\
\hline & Radiação Solar & Câncer de pele \\
\hline & Ruído e Vibrações & $\begin{array}{c}\text { Dor de cabeça, perda parcial ou total da audição, } \\
\text { efeitos adversos na coluna vertebral e outros } \\
\text { membros. }\end{array}$ \\
\hline QUÍMICOS & $\begin{array}{l}\text { Agentes químicos } \\
\text { diversos, tais como: fertilizantes } \\
\text { e adubos, agrotóxicos, gases, } \\
\text { poeira, neblinas e névoas. }\end{array}$ & $\begin{array}{l}\text { Dermatite de contato, rinites, conjuntivite, } \\
\text { intoxicações por agrotóxico, doenças respiratórias } \\
\text { obstrutiva, bronquite, asma ocupacional, doença } \\
\text { pulmonar restritiva, doença pulmonar intersticial com } \\
\text { fibrose, câncer, doença neurológica, alterações do } \\
\text { humor e comportamento, alterações endócrinas, } \\
\text { alterações reprodutivas. }\end{array}$ \\
\hline ERGONÔMICOS & $\begin{array}{l}\text { Posturas incorretas, monotonia e } \\
\text { movimentos repetitivos, } \\
\text { trabalho noturno, ritmos } \\
\text { intensificados de jornada de } \\
\text { trabalho, ausência de pausas } \\
\text { durante o trabalho e treinamento } \\
\text { inadequado. }\end{array}$ & $\begin{array}{l}\text { Sofrimento mental, distúrbios de sono e de humor, } \\
\text { fadiga, Lesão por esforços repetitivos-LER, } \\
\text { Distúrbios osteomusculares relacionados ao trabalho- } \\
\text { DORT. }\end{array}$ \\
\hline BIOLÓGICOS & $\begin{array}{l}\text { Bactérias, vírus, fungos, ácaros, } \\
\text { picadas de } \\
\text { animais peçonhentos. }\end{array}$ & $\begin{array}{l}\text { Rinite, conjuntivite, doenças respiratórias obstrutivas, } \\
\text { asma ocupacional, "pulmão do agricultor" ou } \\
\text { hipersensibilidade ou alveolite alérgica, febre Q, } \\
\text { brucelose, psitaciose, tularemia, tuberculose bovina } \\
\text { ou aviária, leptospirose, histoplamose, raiva, picadas } \\
\text { de cobras e aranhas, queimaduras por lagartas. }\end{array}$ \\
\hline MECÂNICOS & $\begin{array}{l}\text { Ferramentas manuais } \\
\text { cortantes, pesadas, } \\
\text { pontiagudas. }\end{array}$ & $\begin{array}{l}\text { Lesões agudas, acidentes de trabalho } \\
\text { com cortes, esmagamentos, etc. } \\
\text { lesões crônicas, hiperceratose. }\end{array}$ \\
\hline & $\begin{array}{l}\text { Máquinas e implementos } \\
\text { agrícolas. }\end{array}$ & $\begin{array}{l}\text { Acidentes de trabalho, lombalgia, } \\
\text { DORT }\end{array}$ \\
\hline
\end{tabular}

Fonte: Adaptado de SALEM E MOURA (2014); ABREU et al., (2011). 


\section{Ações Propostas}

Órgãos internacionais como a Organização Internacional do Trabalho e a Organização Mundial da Saúde, assim como órgãos competentes de diversos países, divulgam amplamente cartilhas e manuais de como questões não abordadas pela legislação brasileira devem ser levadas em conta. Esses documentos, cujo teor também é divulgado por associações e organizações no Brasil, costumam apresentar em linguagem simples as informações necessárias para o correto uso dos agrotóxicos, alertando para os riscos, bem como instruções para a correta utilização dos EPIs (SALEM; MOURA, 2014).

As ações de melhoria das condições e meio ambiente de trabalho devem abranger os aspectos relacionados aos riscos químicos, físicos, mecânicos e biológicos; investigação e análise dos acidentes e das situações de trabalho que os geraram; e organização do trabalho (BRASIL, 2005).

Entre as bases de dados disponíveis para comunicação de acidentes que envolva a saúde do trabalhador destaca-se: a Comunicação de Acidente do Trabalho (CAT), o Sistema de Mortalidade (SIM), Sistema de Internação Hospitalar (SIH), o Sistema Nacional de Informação Tóxico-Farmacológica (Sinitox) e Sistema Nacional de Informação de Agravos Notificáveis (Sinan).

Os profissionais que atuam na área de ciências agrárias devem estar atualizados quanto ao uso correto dos EPIs, por meio de capacitação e do acesso a informações atualizadas. Bem informado, esses profissionais poderão adotar medidas, cada vez mais econômicas e eficazes, para proteger a saúde dos trabalhadores, além de evitar problemas trabalhistas. (BARROSO E WOLFF, 2009).

Assim sendo, o esclarecimento das condições de risco na qual os agricultores estão expostos, a redução da exposição direta de fertilizantes químicos sem o uso dos EPIs e as melhorias das condições de trabalho compreendem ações capazes de garantir a proteção e redução dos impactos á saúde do trabalhador rural.

\section{CONCLUSÕES}

Através da bibliografia revisada nesta pesquisa, verifica-se que existe uma dificuldade na disseminação do uso dos EPIs no trabalho rural, por diversos motivos, muitos deles relacionados com o treinamento e as condições sociais e ambientais em que estão expostos os trabalhadores.

Assim sendo, o desafio é reduzir os impactos do uso inadequado dos EPIs e do uso de produtos químicos acima da capacidade de suporte do ambiente, em contrapartida fornecer suporte ao melhor desenvolvimento das atividades em campo, a partir das análises dos dados a serem obtidos, com foco na minimização dos riscos na qual os humanos e o meio ambiente são alvos.

\section{REFERÊNCIAS BIBLIOGRÁFICAS}

ABREU, D., MORAES, L. A., NASCIMENTO, E. N., OLIVEIRA, R. A. A Produção de Cana-de-açúcar no Brasil e a Saúde de Trabalhador Rural. Revista Brasileira de Medicina do Trabalho. Ed. 9(2), 2011, p. 49-61.
ANVISA - AGÊNCIA NACIONAL DE VIGILÂNCIA SANITÁRIA. Seminário volta a Discutir Mercado de Agrotóxicos em 2012, Brasília; 2012. Disponível em: <http://portal.anvisa.gov.br> Acesso em: 26 nov. 2014.

APPOLINÁRIO, F. Metodologia da Ciência: filosofia e prática da pesquisa. São Paulo: Pioneira Thomson Learning, 2006. p. 59-70.

AZEVEDO, F. R., FREIRE, F. das C. O. Tecnologia de Aplicação de Defensivos Agrícolas. Documentos Empresa Brasileira de Pesquisa e Agropecuária EMBRAPA, Fortaleza, CE, dez de 2006, 48p.

BARROSO, L. B., WOLFF, D. B. Riscos e Segurança do Aplicador de Agrotóxicos no Rio Grande do Sul. Série: Ciências Naturais e Tecnológicas, S. Maria, v. 10, n. 1, p. 27-52, 2009.

BRASIL. Lei $\mathrm{n}^{\circ} 7.802$ de 11 de julho de 1989. Diário Oficial da União, Brasília, DF, 12 de jul de 1989.

BRASIL. Decreto $\mathrm{n}^{\mathrm{o}} 4.074$ de 04 de janeiro de 2002. Regulamenta a Lei n ${ }^{\circ} 7.802 / 89$. Diário Oficial da União, Brasília, DF, 08 de jan de 2002.

BRASIL. Ministério do Trabalho e Emprego (2005). Normas Regulamentadoras - NR 31. Segurança e Saúde no Trabalho na Agricultura, Pecuária Silvicultura, Exploração Florestal e Aquicultura. Disponível em: <http://www.mte.gov.br/legislacao/normas_regulamentad oras/nr_31.pdf $>$. Acesso em 15 dez. 2014.

CORDEIRO, T. A. O que você precisa saber sobre a água de João Pessoa. Editora Ideia, João Pessoa, 2014, 196 p.

DOMINGUES, E. Sistema de Informação Geográfica e a Contaminação de Agrotóxicos no Meio Ambiente da Cana-de-Açúcar na Bacia Hidrográfica do Rio Pardo SP/MG. 2010. 149f. Tese (Doutorado em Geografia) Instituto de Geociências e Ciências Exatas, Universidade Estadual Paulista, São Paulo, 2010.

IWAMI, A., FERREIRA, C. P., DINNOUTI, L. A., BUENO, F., ARAÚJO, R. M., GOLSALVES, T., SANTIAHO, T. Manual de Uso Correto e Seguro de Produtos Fitossanitários/Agrotóxicos. ANDEF - Associação Nacional de Defesa Vegetal, São Paulo, Linea Creativa, $2^{\circ}$ ed, 2002, 28p.

LINS FILHO, L. A. L. Análise dos Resíduos de Agrotóxicos em Abacaxi: Implicações no Meio Ambiente e Saúde. 2013. 53f. Trabalho de Conclusão do Curso (Graduação em Engenharia Ambiental) - Centro de Tecnologia, Universidade Federal da Paraíba, João Pessoa, 2013.

MONQUERO, P. A., INÁCIO, E. M., SILVA, A. C. Levantamento de Agrotóxicos e Utilização de Equipamento de Proteção Individual entre os Agricultores da Região de Araras. Arq. Inst. Biol., São Paulo, v.76, n.1, p.135-139, jan./mar., 2009. 
PINHEIRO, F. A., ADISSI, P. J. Avaliação de Risco Ocupacional na Aplicação Manual de Agrotóxicos. XXVII Encontro Nacional de Engenharia de Produção, 27. 2007, Foz do Iguaçu. CD ROM ENEGEP. Rio de Janeiro: ABEPRO, 2007.

SALEM, R. E. P., MOURA, L. F. A utilização de equipamentos de proteção individual (EPIs) na manipulação de agrotóxicos: análise sob os aspectos legais e ergonômicos. IV CONGRESSO BRASILEIRO DE ENGENHARIA DE PRODUÇÃO. Ponta Grossa, PR, Brasil, dez de 2014. Anais... Disponível em: <http://www.aprepro.org.br/conbrepro/2014/anais/artigos/ eng\%20t/29.pdf> Acesso em: 23 ago. 2015.

SILVA, J. M.; SILVA, E. N.; FARIA, H. P.; PINHEIRO, T. M. M. Agrotóxico e trabalho: uma combinação perigosa para a saúde do trabalhador rural. Revista Ciência e Saúde Coletiva, v.10 n.4, Rio de Janeiro, out./dez. 2005, 13 p. Disponível em:<http://www.scielo.br/pdf/csc/v10 n4/a13v10n4.pdf >. Acesso em: 10 out. 2015.

VEIGA, M. M., DUARTE, F. J. de C. M., MEIRELLES, L. A., GARRIGOU, A., BALDI, I. A contaminação por agrotóxicos e os Equipamentos de Proteção Individual (EPIs). Revista Brasileira de Saúde Ocupacional. São Paulo, v. 32, p. 57-68, 2007.

VIGNA, C. R. M. Aplicação de Polissiloxanos Imobilizados sobre Sílica como Fase Estacionária e como Sorvente na Determinação de Agrotóxicos em Água e Caldo de Cana. 2010.137 f. Tese (Doutorado em Química). Universidade Estadual de Campinas, Instituto de Química, Campinas-SP, 2010.

WAICHMAN, A. V. Uma proposta de avaliação integrada de risco do uso de agrotóxicos no estado do Amazonas, Brasil. Revista Acta Amazonica, vol. 38(1), p.45-50. 2008. 